\title{
A Small-Size Polarization Splitter Based on a Planar Optical Phased Array
}

\author{
ARJEN R. VELLEKOOP AND MEINT K. SMIT
}

\begin{abstract}
A novel planar polarization splitter based on an optical phased array is proposed and demonstrated. The dispersive properties of an array of bent optical waveguides is utilized for splitting the two polarizations present in the input waveguide. An experimental device for operation at a $633-\mathrm{nm}$ wavelength, with dimensions of $0.6 \times 2.5$ $\mathrm{mm}^{2}$, was designed and fabricated using conventional (high-quality) optical lithography. Insertion losses as low as $0.5 \mathrm{~dB}$ and far-end crosstalk values of 17-21 dB have been achieved.
\end{abstract}

\section{INTRODUCTION}

A PROBLEM in designing coherent optical receivers is that the polarization state of the input signal is arbitrary. This problem may be solved by applying a polarization controller, which adjusts the polarization of the detected signal to that of the local oscillator, using a feedback signal derived from the detected signal. Because of the small magnitude of the Pockels effect in III-V semiconductors, it will be difficult to miniaturize such a controller in a monolithical integrated circuit to any extent. Further, a number of controllers would be required for endless control.

Recent progress in polarization scrambling techniques [1] indicates the possibility of polarization-insensitive coherent detection. The polarization at the transmitter is scrambled with a high frequency. However, the high scrambling frequency imposes a large receiver bandwidth requirement, and the "on-off" effect leads to a 3-dB minimum loss.

Another approach is polarization diversity, i.e., splitting the two polarizations in the receiver and processing them separately. The most important component in a polarization diversity system is the TE-TM polarization splitter.

Several concepts have been proposed for planar polarization splitters. Bergman et al. [2] proposed a splitter based on intersecting waveguides (device length $25 \mathrm{~mm}$ ), which showed a 1.7-dB insertion loss and a 12-15 dB crosstalk. Ura et al. [3] demonstrated a focusing grating coupler with a 4-dB coupling loss, a $14-17-\mathrm{dB}$ crosstalk, and a total device area of $1 \times 11 \mathrm{~mm}^{2}$. Aarnio [4] reported the smallest splitter device made so far. It was also based on a grating coupler (device length $5 \mathrm{~mm}$ ) but with a channel isolation of $9-11 \mathrm{~dB}$. Neyer and Dang [5] reported a $3-\mathrm{dB}$ loss figure and a $20-\mathrm{dB}$ crosstalk for a con-

Manuscript received January 12, 1989; revised June 27, 1989.

The authors are with the Department of Electrical Engineering, Delft University of Technology, Delft, the Netherlands.

IEEE Log Number 8931028 figuration based on two-mode interference (device length $20 \mathrm{~mm}$ ). Mikami [6] achieved loss and crosstalk values of $1.7 \mathrm{~dB}$ and $20 \mathrm{~dB}$, respectively, using a $15-\mathrm{mm}$ long directional coupler. In addition, this device requires a tuning circuit for proper operation.

In this paper, a new planar polarization splitter with a $0.5-\mathrm{dB}$ loss and a $20-\mathrm{dB}$ crosstalk for the TM channel and a 1.7- $\mathrm{dB}$ loss and $17-\mathrm{dB}$ crosstalk for the TE channel is presented. The component, which is based on an optical phased array and is realized by conventional optical lithographic technology, has dimensions of $0.6 \times 2.5$ $\mathrm{mm}^{2}$.

\section{BASIC CONCEPT}

Smit [7] proposed and demonstrated that a properly designed array of concentrically bent waveguides has both focusing and dispersive properties. However, the component had considerable loss (evidenced by the occurrence of multiple foci in the focal plane), which is characteristic in phased arrays. The power coupled to higher order beams can be reduced by spacing the individual elements more closely. This can be achieved by providing the concentric array of a fan-in and fan-out coupling section at both ends, as is shown in Fig. 1. These coupling sections gradually adapt the incoming and outgoing beams to the set of guided modes. To obtain a smooth connection between the concentric sections and the coupling sections, an adapter section is required.

The phase transfer of the complete phased array (including coupling and adapter sections) can be controlled by choosing the radii $R_{i}$ of the concentric section such that the total length of each channel equals an integer number of wavelengths. This choice of the phase transfer will transform the divergent incoming beam into a convergent outgoing one with the same angular intensity distribution so that the source field at the transmitter side will be reproduced in the focal plane at the receiver side. Since the phase transfer is determined by the product of the propagation constant $\beta$ and the total length $l_{i}$ of each channel, a small variation of $\beta$ will result in a variation of the phase transfer, which is proportional to the channel length. If the array is properly designed, the channel length $l_{i}$ will increase linearly with $i$. Consequently, on changing $\beta$, the outgoing wavefront will tilt, thus leading to a lateral shift of the focal position.

Since the propagation constant $\beta$ in a waveguide depends on the polarization as well as the wavelength, the 


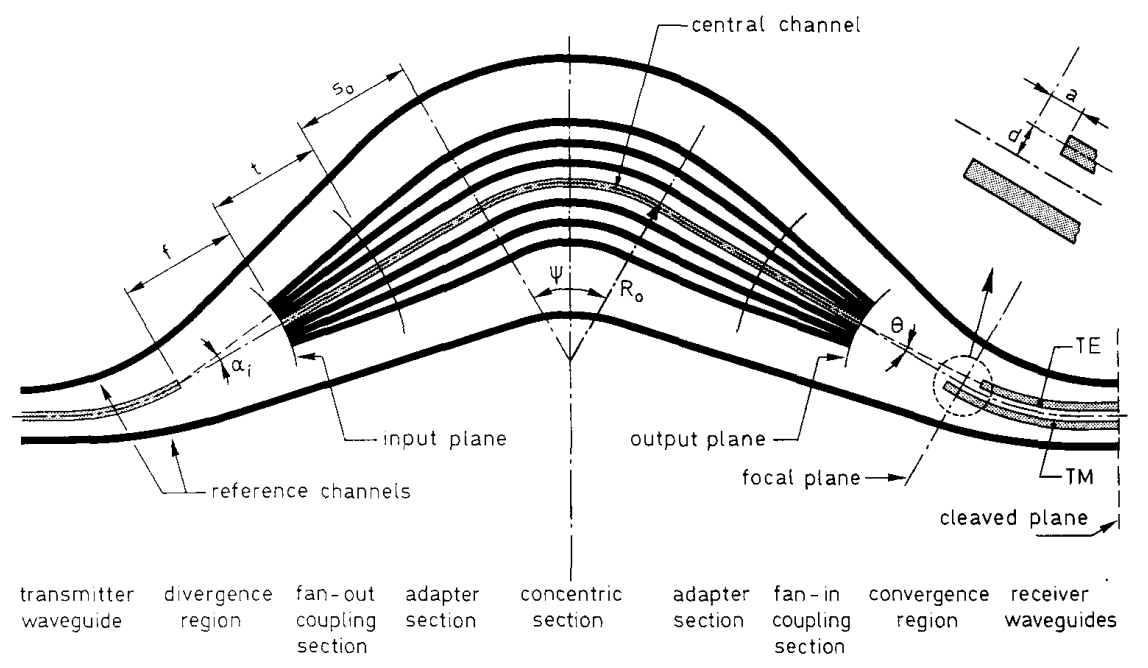

Fig. 1. Polarization splitter geometry with coresponding design parameters (drawing is not to scale).

phased array can, in principle, operate as a polarization splitter as well as a wavelength (de)multiplexer, provided the parameters of the structure are suitably chosen.

\section{Polarization Splitter Analysis}

\section{A. Phase Transfer}

With the array geometry as depicted in Fig. 1, it is easily seen that the phase transfer of the $i$ th channel relative to the central channel follows as

$$
\begin{aligned}
\Phi_{i} & =-\beta \cdot\left(l_{i}-l_{0}\right) \\
& =-\beta \cdot\left\{2 \cdot\left(s_{i}-s_{0}\right)+\Psi \cdot\left(R_{i}-R_{0}\right)\right\}
\end{aligned}
$$

in which $\Psi \cdot\left(R_{i}-R_{0}\right)$ is the difference in length between the $i$ th and the central channel of the concentric section, and $2 \cdot\left(s_{i}-s_{0}\right)$ is the same difference for the two adapter sections. To obtain focusing action, the radii $R_{i}$ are chosen equidistant in principle and rounded to the value for which the relative phase transfer $\Phi_{i}$ equals an integer multiple of $2 \pi$. The solution of $R_{i}$ from (1) is discussed in more detail in Appendix A.

\section{B. Dispersion and Aberration}

Each channel of the phased array can be characterized by the coordinate $\alpha_{i}$ of its starting point at the transmitter side ( $\alpha_{i}$ being the angular coordinate in a polar coordinate system, as depicted in Fig. 1). The relative channel length $l_{i}-l_{0}$ can be developed in a Taylor series as (Appendix A):

$$
\begin{aligned}
& \left(l_{i}-l_{0}\right)=\psi \cdot\left(f+t+\frac{s_{o}}{2}\right) \cdot \alpha_{i}+\left(f+t+\frac{s_{0}}{3}\right) \\
& \cdot \alpha_{i}^{2}+\frac{1}{6} \cdot\left(f+t+\frac{s_{0}}{2}\right) \cdot \alpha_{i}^{3}+0\left(\alpha_{i}^{4}\right)
\end{aligned}
$$

in which $f$ is the focal length, $t$ is the length of the fan-in and fan-out sections, and $s_{0}$ is the central-channel length of the adapter section. Through a proper choice of the $R_{i}$ 's, the array is designed such that $\Phi_{i}\left(\beta_{0}\right)=0$ for all $i$. Consequently

$$
\Phi_{i}(\beta)=-\left(\beta-\beta_{0}\right) \cdot\left(l_{i}-l_{0}\right) .
$$

The phase-transfer function $\Phi_{i}(\beta)$ thus contains linear, quadratic, and higher order terms in $\alpha_{i}$. The linear term describes the dispersion of the array. The tilting angle $d \theta$ of the outgoing wavefront is determined by the tangent of the phase difference between two adjacent channels, which is expressed as a fraction of the wavelength $(\Delta \Phi / \beta)$, divided by the distance $f \cdot \Delta \alpha$ between the channels according to

$$
\frac{d \theta}{d \beta} \simeq \frac{\frac{d}{d \beta} \cdot\left(\Phi_{i+1}-\Phi_{i}\right)}{\beta f \cdot\left(\alpha_{i+1}-\alpha_{i}\right)}=\frac{-1}{\beta^{2} f} \frac{d \Phi}{d \alpha} .
$$

At the receiver end, this angular tilt will cause a lateral displacement of the focal point

$$
d=f \cdot \frac{d \theta}{d \beta} \cdot \Delta \beta=\frac{\Delta \beta}{\beta} \cdot \Psi \cdot\left(f+t+\frac{s_{0}}{2}\right) .
$$

For the waveguide structure as described in Section IV, which is operated at $633 \mathrm{~nm}$, the relative difference between $\beta_{\mathrm{TE}}$ and $\beta_{\mathrm{TM}}$ is approximately 1.3 percent. If the polarization splitter is designed to have zero dispersion at the center value $\beta_{0}=\frac{1}{2}\left(\beta_{\mathrm{TE}}+\beta_{\mathrm{TM}}\right), \Delta \beta / \beta$ becomes \pm 0.65 percent for the TE and the TM channel, respectively.

With $f=100 \mu \mathrm{m}, t=100 \mu \mathrm{m}, s_{0}=190 \mu \mathrm{m}$, and $\Psi$ $=1.3 \mathrm{rad}$, which are representative values for a splitter design as discussed in Section IV, the dispersion angle $\theta_{\mathrm{TE}, \mathrm{TM}}= \pm 1.5^{\circ}$ and the corresponding dispersive displacement $d= \pm 2.5 \mu \mathrm{m}$. This means that the distance between the TE and TM receiver waveguides thus becomes $5 \mu \mathrm{m}$. 
The dispersive properties of the splitter also apply to wavelength variations so that a wavelength shift $\Delta \lambda$ will decrease the splitter performance. As long as these variations are within \pm 10 percent of the relative difference $\left(\beta_{\mathrm{TE}}-\beta_{\mathrm{TM}}\right) / \beta_{o}$, the effect will be small. With the above values, the acceptable wavelength fluctuation $\Delta \lambda$ is approximately $\pm 1 \mathrm{~nm}$. For longer wavelengths ( 1.3 and $1.55 \mathrm{~nm}$ ) and higher contrast, the wavelength stability requirements will become less critical.

At the central propagation constant $\beta_{0}$ the wavefront curvature is $1 / f$. With varying propagation constant, the second-order term of (2) introduces an additional phase difference between adjacent channels proportional to the square of the angular coordinate of the $i$ th channel $\left(\alpha_{i}^{2}\right)$. This phase difference leads to an additional wavefront curvature $\Delta c$, which is described by the second derivative of $\Phi$. It can be inferred that this extra curvature is described by

$$
\frac{d c}{d \beta}=\frac{-1}{f^{2}} \cdot \frac{d^{2} \Phi / d \alpha^{2}}{\beta^{2}}
$$

The corresponding change in the focal length is

$$
\frac{d f}{d \beta}=\frac{d(1 / c)}{d \beta}=-f^{2} \cdot \frac{d c}{d \beta}=\frac{1}{\beta^{2}} \frac{d^{2} \Phi}{d \alpha^{2}} .
$$

The aberration $a$ is thus found as

$$
a=\frac{d f}{d \beta} \cdot \Delta \beta=2 \cdot \frac{\Delta \beta}{\beta} \cdot\left(f+t+\frac{s_{0}}{3}\right) .
$$

For small values of $s_{0}$, the ratio $d / a$ approaches $\frac{1}{2} \Psi$. In the above example, the aberration $a_{\mathrm{TE}, \mathrm{TM}}$ of the two channels thus becomes $\pm 3.4 \mu \mathrm{m}$.

\section{Insertion Loss}

The field intensity distribution in the focal plane is determined by the multiplication of the array function of the phased array (Fig. 2, pattern $b$ ), with the angular radiation pattern of the singular waveguides (Fig. 2, pattern $a$ ) leading to a decreasing field intensity next to the optical axis (Fig. 2, pattern $c$ ). To estimate the corresponding loss, the mode profile of the singular waveguides is approximated as $I(x) \simeq \exp \left(-2 x^{2} / w_{0}^{2}\right)$, where the effective beam waist $w_{0}$ is computed from $w_{0}=2 / \sqrt{\pi} \cdot \int$ $I(x) d x$, and where the integral is being taken from 0 to $\infty$. The radiation pattern of such a Gaussian profile follows as

$$
I(\theta)=\left[e^{-\theta^{2} / \theta_{0}^{2}}\right]^{2}
$$

in which the effective beam width $\theta_{0}=\lambda / \pi w_{0}=2 / \beta w_{0}$ ( $\lambda$ is the wavelength in the film).

The dispersion angle $\theta_{\max }$ corresponding to the maximum acceptable loss $L_{\max }$ thus follows as

$$
\theta_{\max } \simeq \theta_{0} \sqrt{\left(\frac{L_{\max }}{20 \cdot \log (e)}\right)}
$$

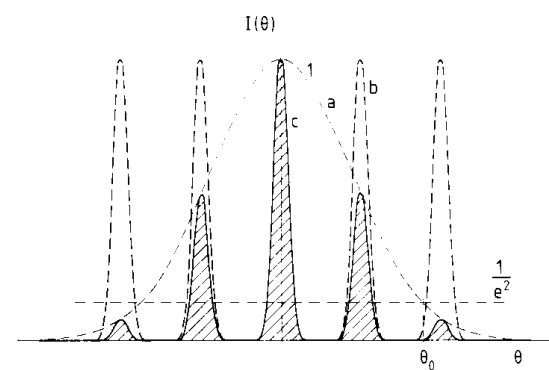

Fig. 2. Field intensity distribution $I$ as a function of the dispersion angle $\theta$. The field intensity (pattern $c$ ) is formed by the product of the angular radiation pattern of the singular waveguides (pattern $a$ ), and the array function of the phased array (pattern $b$ ).

For the waveguides described in Section IV, $\theta_{0}$ becomes approximately $6^{\circ}$. With a maximum acceptable channel loss $L_{\max }=0.5 \mathrm{~dB}$, the dispersion angle $\theta_{\max }$ becomes $1.5^{\circ}$.

\section{Channel Crosstalk}

Small derivations in the transfer characteristics of the array from the ideal case will predominantly affect the channel isolation properties and will have less of an effect on the insertion loss. There are a number of mechanisms degrading the channel isolation, and these will be discussed as follows:

1) If the receivers are placed close to each other, part of the receiver power in the focal plane will not only couple to the intended receiver waveguide but also to the adjacent one. Because the field distribution in the focal plane is the optical image of the transmitter mode profile, this effect is easily estimated by taking the overlap integral of the fundamental modes of the adjacent waveguides. An expression for this mechanism is derived in Appendix B. For the waveguides described in Section IV and a waveguide spacing of $5 \mu \mathrm{m}$, a channel isolation of $-60 \mathrm{~dB}$ is predicted.

2) Adjacent waveguides in the fan-in and fan-out sections will exhibit some mutual coupling. Because the waveguides in the fan-out section are excitated with the same phase and only a slightly different amplitude, there will be negligible mutual coupling. Once it arrives at the fan-in section, the light has travelled through channels of different lengths. Consequently, the modes in the different waveguides will have different phases, and some mutual coupling that distorts the output plane phase distribution will occur. This mechanism will be negligible for most applications, as is shown in Appendix C.

3) Due to the higher order terms in (3), a degradation of the focal field will occur, thus leading to an increased crosstalk between the two different receiver channels. It is seen from (2) that the contribution of the third and higher order terms is approximately a fraction $\frac{1}{6} \cdot \alpha^{2}$ of the linear term. Even at the ex- 
treme ends of the array, this contribution will generally be less than 1 percent.

4) Transitions between straight and bent waveguides, as well as waveguide imperfections, may cause partial conversion of the fundamental mode to higher order lateral modes. If these modes, with different propagation constants, are guided by the array waveguides, which is the case for the bimodal waveguides applied in the present design, they will distort the phase transfer. Similar distortion will occur in the case of polarization conversion at waveguide imperfections

5) Random fluctuations in the waveguide properties (width, thickness, refractive index), will produce local variations in the propagation constant $\beta$ and will consequently lead to random phase fluctuations in the output plane. These phase fluctuations produce background radiation in all directions, thus leading to channel crosstalk.

The two latter effects, which are due to stochastic imperfections in the waveguides, can only be determined experimentally.

\section{Polarization Splitter Design}

Because an accurate measurement configuration operating at a $633-\mathrm{nm}$ wavelength is available, the experimental polarization splitter was designed for this wavelength. However, using the same principles mentioned before, polarization splitters operating at longer wavelengths (e.g., 1.3 or $1.55 \mu \mathrm{m}$ ) can easily be designed.

An important issue in designing the polarization splitter is the choice of the waveguide width and the optical contrast. A small waveguide width $w$ allows for a close spacing of the receiver waveguides. Through (5), this leads to a small value of $f, t$, and $s_{0}$ and, consequently, a small device size. The minimal waveguide width is determined by the geometrical reproducibility of the lithographic process. A compromise was found in a waveguide width of $2 \mu \mathrm{m}$.

A high effective-refractive-index contrast allows for a small radius of curvature but causes the waveguide to become multimode and the waveguide attenuation to increase. A compromise was found in a bimodal waveguide structure with a contrast $\Delta n=0.02$, which combines a waveguide attenuation of $2 \mathrm{~dB} / \mathrm{cm}$ with negligible radiation loss for radii greater than $700 \mu \mathrm{m}$. The computations were based on the work of Pennings et al. [8].

The splitter was designed on an insertion loss $L_{\max }$ of $0.5 \mathrm{~dB}$, which occurs with the above waveguide parameters at a dispersion angle of $1.5^{\circ}(10)$. With this value of the dispersion angle, a focal length $f$ of $100 \mu \mathrm{m}$ is required to arrive at a dispersive displacement of $d=2.5$ $\mu \mathrm{m}$. As shown in Appendix $B$, the predicted channel isolation for a channel separation of $5 \mu \mathrm{m}$ is $-60 \mathrm{~dB}$.

The adjacent waveguides in the fan-in and fan-out sections will exhibit some mutual coupling. In Appendix C. it is shown that this coupling is negligible if the length $l$ is chosen the same as the focal length $t=f=100 \mu \mathrm{m}$.
TABLE I

Design Parameters and Theoretical Properties of the Polarization SPLITTER

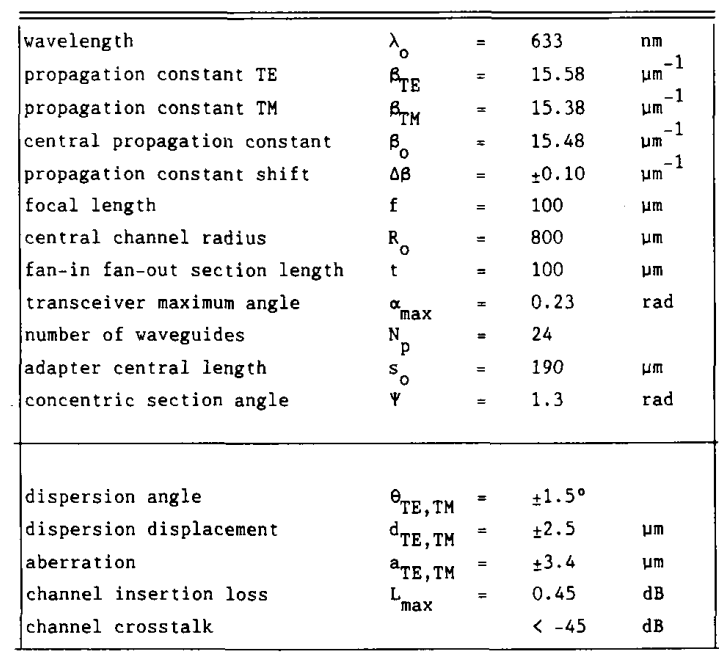

The number of waveguides $N_{p}$ determines the aperture width of the phased array. Due to the finite width of the array aperture, the field distribution at the output plane will be truncated and side lobes, which cause channel crosstalk, will occur in the focal plane. For a rough estimate of the side-lobe levels, it is noted that the power in the side lobes will be of the same order as the power in the truncated part of the field distribution. This power is equal to the spillover at the input plane. If $\alpha_{\max }$ is chosen to be twice the effective beam width $\theta_{0}$, the spillover is estimated to be less than 0.01 percent. In the worst case, all this power is coupled into the adjacent channel so that the channel crosstalk will be $-40 \mathrm{~dB}$ at the most. For the $2-\mu \mathrm{m}$ waveguides chosen, $\theta_{0}=6^{\circ}$. With $f=100 \mu \mathrm{m}$, this leads to $N_{p}=24$.

To avoid radii smaller than $700 \mu \mathrm{m}$ at the lower part of the concentric section (see Fig. 1), $R_{0}$ is chosen to be 800 $\mu \mathrm{m}$. To connect the fan-in and fan-out sections smoothly with the concentric section, the adapter section contains two circular waveguide sections with slightly different radii. If the central adapter section length $s_{0}$ is chosen too small, the radius of one of the circular sections may become smaller than $700 \mu \mathrm{m}$. From Appendix A, it follows that this will not happen if $s_{0} \geq R_{0} \sin \alpha_{\max }$. A value of $s_{0}=190 \mu \mathrm{m}$ is found to be sufficient.

Once $f, t$, and $s_{0}$ are determined, the concentric-section sector angle follows from (5). With a relative propagation constant shift $\Delta \beta / \beta=0.65$ percent, this angle becomes $\Psi=1.3 \mathrm{rad}$.

Table I summarizes the design parameters and the corresponding theoretical properties of the phased array.

\section{Simulation}

A numerical simulation can be performed by following the transmitted signal through the device. First, the lateral fundamental-mode profile is calculated in the transmitter 


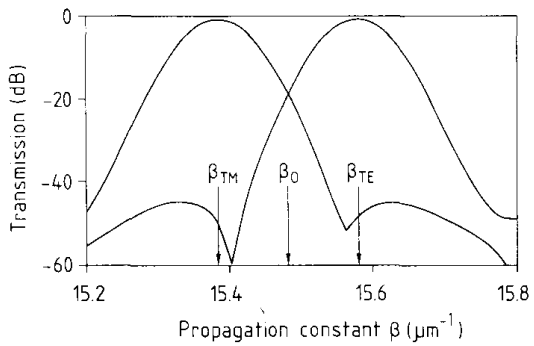

Fig. 3. Numerically simulated power transfer for the TE and the TM channel as a function of the propagation constant $\beta$. The design values for $\beta_{\mathrm{TE}}$ and $\beta_{\mathrm{TM}}$ are indicated.

waveguide using the effective-index method. Next, the two-dimensional diffraction field in the input plane of the phased array is determined. The coupling of this field to the guided modes of the array is computed by taking the overlap integral of this input field with the sum field of the individual modes in the input plane of the array. To compute this sum field, it is assumed that the excitation coefficients of the individual channels are proportional to the amplitude of the excitating field. The propagation through the array is described by $\exp \left(-j \beta l_{i}\right)$, where $l_{i}$ is the total channel length. At the output plane, the sum field of the individual channels now becomes the source field.

Finally, the two-dimensional diffracted field in the focal plane is calculated. This field is overlapped with the modal fields of the receiver waveguides to compute the power coupled to the different receiver channels.

The coupling at the receivers and the higher order terms in (2) (Sections III-D-1 and III-D-3) are inherently accounted for in this simulation. The mutual coupling is negligible (Appendix C), whereas the stochastic imperfections can only be determined experimentally. Fig. 3 shows the power coupled to the TE and TM channel as a function of $\beta$. The lobes occurring below $-45 \mathrm{~dB}$ are caused by the finite aperture of the phased array and are in good agreement with the rough estimate made in Section IV. As can be seen from Fig. 3, the predicted insertion loss and channel isolation at the values of $\beta$ corresponding to $\beta_{\mathrm{TE}}$ and $\beta_{\mathrm{TM}}$ are approximately $-0.5 \mathrm{~dB}$ and $-50 \mathrm{~dB}$, respectively.

\section{FABRICATION}

The experimental devices were realized using a $\mathrm{SiO}_{2} / \mathrm{Al}_{2} \mathrm{O}_{3} / \mathrm{SiO}_{2}$ waveguide structure on a silicon substrate. The waveguides wera fabricated by RF-sputter depositing a $0.25-\mu \mathrm{m} \mathrm{Al} \mathrm{O}_{3}$ film $(n \simeq 1.69)$ onto a thermally oxidized silicon substrate, as described by Smit $e t$ al. [9], [10]. The lateral waveguide structure is produced by atom-beam milling a $40-\mathrm{nm}$ step in this layer through a photo-resist pattern. The $2-\mu \mathrm{m}$ waveguide pattern is obtained by projecting a chromium mask, which is created by an optical pattern generator (ASET COMBO 250), onto an image-reversal resist film (Hoechst 5214 E) with a $4 \times$ Canon reduction camera (FPA 141). The etched structure is covered with a $0.6-\mu \mathrm{m}$ RF-magnetron sputtered $\mathrm{SiO}_{2}$

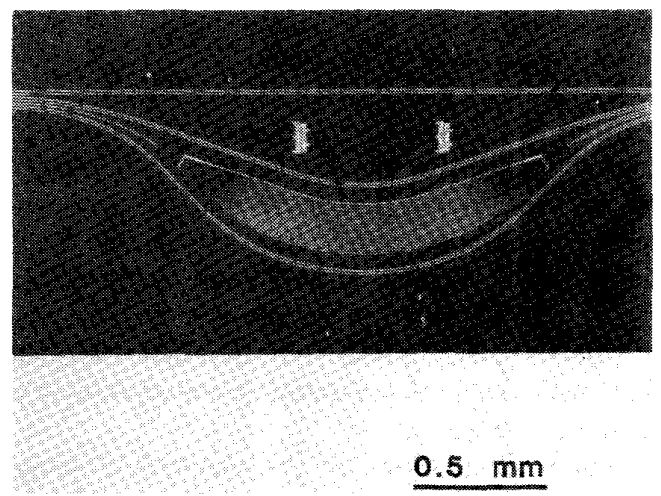

Fig. 4. Photograph of the polarization splitter. The two bent waveguides at each side of the phased array and the two straight waveguides are reference channels.

layer $(n \simeq 1.46$ ) so that an embedded ridge-guide structure is obtained.

Fig. 4 shows a photograph of the experimental device. The two bent waveguides at each side of the phased array and the two straight waveguides are used as reference channels. By comparing the simultaneously excitated reference channels with the receiver channels, loss and crosstalk values can be determined.

\section{EXPERIMENTAL RESUlts}

The experimental device was investigated by selectively coupling TE- or TM-polarized light from an He-Ne gas laser operating at $633 \mathrm{~nm}$ into the planar waveguides with a prism coupler, as described by Pasmooij et al. [11]. The device was cleaved at the position indicated in Fig. 1. The endface of the device is projected onto a CCD video camera with a microscope objective. The signal from the camera is digitized and processed by a computer. Fig. 5 shows photographs of the camera observations for the two polarizations. An intensity scan over the different channels is shown in Fig. 6.

Insertion loss can be determined by comparing the intensity of the receiver channel with the intensities of the reference channels, as is shown in Fig. 6. Table II lists the values measured for four different devices. The values measured for devices 3 and 4 are less representative due to a poor match of the required values of $\beta_{\mathrm{TE}}$ and $\beta_{\mathrm{TM}}$. From the listed values, it is clear that an insertion loss close to the theoretically predicted value of $0.5 \mathrm{~dB}$ is obtained for the TM channel (device 2). The extremely low values listed in Table II are most probably due to measurement errors, which are estimated to be within \pm 0.5 $\mathrm{dB}$. The higher loss for the TE channel is not yet understood.

Channel isolation is determined by comparing the transmitted signal intensity for a single polarization with the signal level in the other channel after removing a 20 -dB optical attenuator. The crosstalk values of $17-20$ $\mathrm{dB}$ (devices 1 and 2 ) are worse than the theoretical predictions but are comparable with results published so far. Stochastic waveguide imperfections (Sections III-D-4 and 
(a)

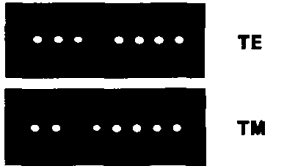

Fig. 5. Camera observations of the channels at the cleaved-end face of the device for (a) TE polarization and (b) TM polarization.

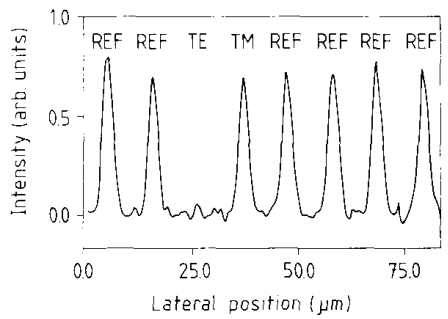

Fig. 6. Intensity scan of the TM polarization. Estimated insertion loss is $0.25 \mathrm{~dB}$.

TABLE II

Measurement Results of Four Different Devices. Crosstalk Values Are Determined in Relation to the Other Receiver Channel. In the Second Colunin, the Measured Mismatch of the Experimental. Device Relative to the Design Value Is Indicated

\begin{tabular}{|c|c|c|c|c|c|}
\hline \multirow{2}{*}{ Nr. } & \multirow{2}{*}{ mismatch } & \multicolumn{2}{|c|}{ TB } & \multicolumn{2}{|c|}{ TM } \\
\cline { 3 - 6 } & $\Delta B / \beta(\%)$ & $\begin{array}{c}\text { loss } \\
\mathrm{dB}\end{array}$ & $\begin{array}{c}\text { crosstalk } \\
\mathrm{dB}\end{array}$ & $\begin{array}{c}\text { loss } \\
\mathrm{dB}\end{array}$ & $\begin{array}{c}\text { crosstalk } \\
\mathrm{dB}\end{array}$ \\
\hline 1 & 0.03 & 2.0 & -21.1 & 2.0 & -19.3 \\
2 & 0.05 & 1.7 & -17.0 & 0.3 & -20.2 \\
3 & 0.12 & 1.5 & -17.6 & 0.1 & -13.3 \\
4 & 0.12 & 2.1 & -19.0 & 0.3 & -17.0 \\
\hline
\end{tabular}

III-D-5) may be responsible for this performance degradation. Results of research on these effects will be published in future work.

\section{CONCLUSIONS}

A new polarization splitter based on an optical phased array is reported. A number of experimental devices were realized using conventional (high-quality) optical lithography with $0.5-1.5-\mathrm{dB}$ insertion loss and $17-20-\mathrm{dB}$ crosstalk. These results are comparable with the best results reported so far, but they have been achieved with a considerably smaller device size $\left(0.6 \times 2.5 \mathrm{~mm}^{2}\right)$.

\section{Appendix A}

\section{The Phase Transfer}

The individual waveguides of the fan-in and fan-out coupling section can be described by their angular deviation $\alpha_{i}$ relative to the central channel (Fig. 1) with $\pm \alpha_{\max }$ being the relative angles of the extreme channels. The $i$ th waveguide of the fan-in section is smoothly connected to the concentric section by an arc over an angle $\alpha_{i}$ and with a radius

$$
\begin{aligned}
\rho_{i} & =\frac{s_{0}+(f+t) \cdot\left(1-\cos \alpha_{i}\right)}{\sin \alpha_{i}} \\
\alpha_{i} & =\left\{i-\frac{1}{2}\left(N_{p}+1\right)\right\} \cdot d_{p} / f \quad 1 \leq i \leq N_{p}
\end{aligned}
$$

in which $s_{0}$ is the length of the central channel in the adapter section, $f$ is the focal length, $t$ is the fan-in section length, $w$ is the waveguide width, and $N_{p}$ is the number of waveguides. The radius of the corresponding channel in the concentric section is

$$
R_{i}=R_{0}+2 \cdot\left(f+t+\frac{s_{0}}{2}\right) \cdot \tan \frac{1}{2} \alpha_{i}
$$

where $R_{0}$ is the radius of the central channel.

The total relative length $l_{i}-l_{0}$ of the $i$ th channel thus becomes

$$
l_{i}-l_{0}=\Psi \cdot\left(R_{i}-R_{0}\right)+2 \cdot\left(\alpha_{i} \cdot \rho_{i}-s_{0}\right) .
$$

The development of (A4) in a Taylor series in $\alpha_{i}$, after substitution of (A1) and (A3), yields the expression of (2). To meet the phase-transfer requirement for the $i$ th channel, $R_{i}$ has to be changed by a small amount $d R_{i}$ (to be calculated further on). This change $d R_{i}$ introduces a connection error between the adapter section and the concentric section. This error can be prevented by dividing the adapter section into two circular waveguide sections (each over an angle $\frac{1}{2} \alpha_{\max }$ ) with radii

$$
\begin{aligned}
& \rho_{i 1}=\rho_{i}-\Delta \rho_{i} \\
& \rho_{i 2}=\rho_{i}+\Delta \rho_{i}-d R_{i}
\end{aligned}
$$

in which

$$
\Delta \rho_{i}=\frac{d R_{i}}{2-\sqrt{\left(2+2 \cdot \cos \alpha_{i}\right)}} \quad\left(\alpha_{i} \neq 0\right) .
$$

If $\alpha_{i}=0$, then $d R_{i}=0$ and the adapter waveguide becomes straight. The length $s_{i}$ of the $i$ th channel in the adapter section follows as

$$
s_{i}=\alpha_{i} \cdot\left(\rho_{i}-\frac{1}{2} \cdot d R_{i}\right)
$$

so that the corresponding total relative length becomes

$$
\begin{aligned}
l_{i}-l_{0}= & \Psi \cdot\left(R_{i}-R_{0}\right) \\
& +2\left(\alpha_{i} \cdot \rho_{i}-s_{0}\right)-d R_{i} \cdot(\Psi+\alpha) .
\end{aligned}
$$

To obtain focusing action, the $d R_{i}$ have to be chosen such that $\left(l_{i}-l_{0}\right)$ equals an integral number of wavelengths. If the solution is chosen closest to zero for the $d R_{i}, R_{i}$ will become nonlinear in $\alpha_{i}$, as can be seen from (A3). Because the tangent of $\alpha_{i}$ is described by $\alpha_{i}+\frac{1}{3} \cdot \alpha_{i}^{3}+$ $0\left(\alpha^{5}\right)$, this nonlinearity can be avoided by choosing for $d R_{i}$ the value closest to $\frac{1}{12} \cdot\left(f+t+\frac{1}{2} s_{0}\right) \cdot \alpha^{3}$.

\section{APPENDIX B}

\section{Mode Coupling in the Receiver Channels}

At the receiver, the channel isolation is determined by the overlap of the modes in the receiver channels. The channel isolation follows from the overlap integral

$$
\eta=\frac{\left\{\int U(x) \cdot U\left(x-d_{c}\right) d x\right\}^{2}}{\left\{\int U^{2}(x) d x\right\}^{2}}
$$


in which $d_{c}$ is the distance between the center lines of the receiver waveguides, and $U(x)$ is the lateral mode profile in the waveguides. Substitution of the expressions for $U(x)$ into (B1) yields the following results:

$$
\begin{aligned}
\eta= & {\left[\frac{2 \cdot(1-b) \cdot \exp \left[-V \cdot\left(d_{c}-w\right) \cdot \sqrt{(b)} / w\right]}{1+2 /(V \cdot \sqrt{b})}\right.} \\
& \left.\cdot\left\{\frac{\left(d_{c}-w\right)}{w}+\frac{4 \cdot \sqrt{b}}{V}\right\}\right]^{2}
\end{aligned}
$$

in which $w$ is the waveguide width, $V$ is the normalized frequency, and $b$ is the normalized propagation constant as described by Unger [12].

For the waveguide structure described in Section IV, $V$ and $b$ have the values 4.7 and 0.78 , respectively. With $w$ $=2 \mu \mathrm{m}$ and $d_{c}=5 \mu \mathrm{m}$, the channel isolation becomes $10 \cdot \log \eta<-60 \mathrm{~dB}$.

\section{APPENDIX C}

\section{Mutual Coupling Between Two Waveguides}

To obtain a rough estimate of the coupling effects in the fan-in section, the coupling between two adjacent waveguides is considered using the weak-coupling approach. The mutual coupling between two adjacent waveguides depends on the distance between those waveguides and the waveguide properties. For weak coupling, the coupling coefficient can be described as [12]

$$
c(z)=K \cdot e^{-v \cdot a(z) / w}
$$

with

$$
K=\frac{2 \cdot v u^{2}}{\beta \cdot w^{2} \cdot\left(u^{2}+v^{2}\right) \cdot(1+2 v)}
$$

in which $u$ and $v$ are the normalized transverse propagation coefficients, $\beta$ is the propagation constant, $w$ is the waveguide width, and $a(z)$ is the $z$-dependent distance between the two waveguides. This distance is proportional to $z$ in the phased array according to

$$
a(z)=\frac{w}{f} \cdot z
$$

The cumulative coupling effect over a length $t$ of the fanin section follows from the integral of (C1) as

$$
\begin{aligned}
I(t) & =\int_{0}^{t} K \cdot e^{(-v / f) \cdot z} d z \\
& =\frac{f \cdot K}{v} \cdot\left(1-e^{(-v / f) \cdot t}\right) .
\end{aligned}
$$

It is seen that the contribution to the integral for $z>t$ is negligible ( $<2$ percent) if $(v / f) \cdot t \geq 4$. With $v=V$ - $\sqrt{b}$, where $V$ and $b$ are the normalized frequency and propagation constant with values 4.7 and 0.78 , respectively, the corresponding length $t$ approximately equals $f$.

Substitution of these values into (4) yields $I(\infty)=(f$ - $P(v) \simeq 0.0032$. The power transfer to the adjacent waveguide is described by $\sin ^{2}\{I(t)\}$, and it follows that the counling effects in the fan-in section are negligible.
Although the computations were based on the weak-coupling approximation, which fails close to the output plane, it is believed that the results are indicative for the magnitude of the effects.

\section{ACKNOWLEDGMENT}

The authors wish to thank J. W. M. van Uffelen and A. H. de Vreede for fabricating the devices and Prof. G. A. Acket for his help and useful suggestions.

\section{REFERENCES}

[1] T. G. Hodgkinson, R. A. Harmon, and D. W. Smith, "Polarisationinsensitive heterodyne detection using polarisation scrambling," Electron. Lett., vol. 23, no. 10, pp. 513-514, May 1987.

[2] E. E. Bergmann, L. McCaughan, and J. E. Watson, "Coupling of intersecting $\mathrm{Ti}: \mathrm{LiNbO} 3$ diffused waveguides," Appl. Opt., vol. 23, no. 17 , pp. 3000-3003, Sept. 1984.

[3] S. Ura, H. Sunagawa, T. Suhara, and H. Nishihara, "Focussing grating couplers for polarization detection," J. Lightwave Technol., vol. 6, no. 6, pp. 1028-1033, June 1988.

[4] J. Aarnio, "An integrated-optic polarization splitter on silicon substrate,' in Proc. ECOC' 88 (Brighton, England), Sept. 11-15, 1988, pp. $37-40$.

[5] A. Neyer and D. Dang, "Guided-wave polarization splitter based on two-mode-interference," in Tech. Dig. Integrated and Guided-Wave Optics, Santa Fe, NM, Mar. 28-30, 1988, pp. 107-110.

[6] O. Mikami, "LiNbO3 coupled-waveguide TE/TM mode splitter," Appl. Phys. Lett., vol. 36, no. 7, pp. 491-493, Apr. 1980.

[7] M. K. Smit, "A new focussing and dispersive planar component based on an optical phased array," Electron. Lett., vol. 24 , no. 7 , pp. 385386, Mar. 1988.

[8] E. C. M. Pennings, G. H. Manhoudt, and M. K. Smit, "Low-loss bends in planar optical ridge waveguides," Electron. Lett., vol. 24, no. 16 , pp. 998-999, Aug. 1988.

[9] M. K. Smit, C. J. van der Laan, and G. A. Acket, "Al203-films for integrated optics,"' Thin Solid Films, vol. 138, pp. 171-181, 1986.

[10] M. K. Smit, "Sputtered planar optical waveguides," in Proc. Sensors Actuators (Twente Univ. Tech. Enschede, the Netherlands), Oct. 30-31, 1986, pp. 45-55.

[11] W. A. Pasmooij, M. K. Smit, and P. A. Mandersloot, "Selective and stable excitation of planar optical waveguide modes with the prism coupler," J. Lightwave Technol., to be published.

[12] H. G. Unger, Planar Optical Waveguides and Fibres. Oxford, England: Clarendon, 1977.

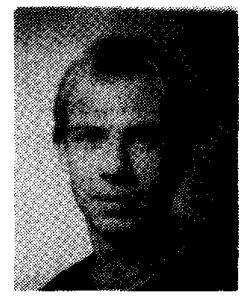

Arjen R. Vellekoop was born in Noordwijkerhout, the Netherlands, on April 11, 1963. He started his study in electrical engineering at the Delft University of Technology, Delft, the Netherlands, in 1981. He has, since 1986, also studied aerospace engineering. He commenced his master's thesis research in 1987 , concentrating on the realization of a polarization splitter and a wavelength demultiplexer based on a planar optical phased array.

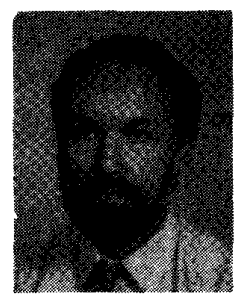

Meint K. Smit was born in Vlissingen, the Netherlands, on January 5, 1951. He graduated, in 1974, from Delft University of Technology, Delft, the Netherlands in electrical engineering.

From 1974 to 1981 , he worked in the field of radar remote sensing first as a Research Assistant at the NIWARS, and since 1976 as a Staff Member at Delft University. Since 1981, he has worked in the field of integrated optics with an emphasis on passive optical components on silicon substrates for use in optical communication. Since 1986 , he has also been engaged in research on the realization of integrated optical circuits in InGaAsP/InP. 